\title{
Seeing Matters: The Remarkable Relevance of the Object-Representation Relationship to Science ... and to Society!
}

\begin{abstract}
The basic relationship between consciously-experienced representations, and material objects they represent, is hotly debated in some circles. But is it practically important? To investigate this, I introduce new symbolic notation, capable of labelling object, brain-perception, and conscious representation. Simple physics-based reasoning argues against identity of object and representation (rejecting e.g., direct realism). Nevertheless, a pivotal concern of the direct-realism school remains: how do we have knowledge of the world, if it's only experienced indirectly? I sketch an indirect-school response, and review recent theoretical results showing how it simply doesn't work in the dynamicallyconventional setting (which is the hallmark of modern mainstream science). After illustrating how dynamically-conventional dysfunctions affect the foundations of science itself, I point to an experimentally-based resolution of knowledge-problems (and of the direct/indirect debate itself). Because the foundational problems for science affect its standing in society (for example, in its conflict with postmodernist 'post-Truth'), the object-representation debate does turn out to have a practical significance, far beyond its conventional, academic/abstract/technical, framing.
\end{abstract}

\section{The Basic Issue: Distal Object, Conscious-Experiential Representation}

Consider a well-defined object in your immediate, visually-experienced, surroundings - a tree, say. Is the appearing constellation of colors, edges, and shape the same thing as the collection of atoms and molecules that make up the tree? Or is it a re-presentation - a sort of three-dimensional picture of the matter-tree, a picture which depends crucially on the brain-activity that can be thought of as encoding the colors, edges, shape, and location of the tree (amongst other things)? For the purposes of this article, I call these two possibilities direct and indirect realism, respectively, and take the competition between these two possibilities as the central question of the present volume. [This anthology limits conscious experience to the visual modality. Some technical issues arise in extending this Chapter's arguments to multi-sensory settings, but these don't compromise overall reasoning (Rosseinsky, 2021a).]

So, indirect realism means that the visual tree-experience indirectly re-presents or resembles the matter-based reality of a tree. [According to this stance, conscious experience is genuinely a representation, because the tree presents itself first as a matter-object. Then the color/edges/shapecomplex is generated as a second kind of presentation, in conscious experience. Contrary to historic argument (Kenny, 1971), there is no 'homunculus' problem for indirectism (French, 2018; Rosseinsky, 2021a).] Ontologically, the indirect position doesn't need to propose matter as a sort of substance-basis 
for reality. (Although it can!) For example, the tree - and all matter - could in principle exist as numbers in a computational reality (Wheeler, 1989). One further step hypothesizes that this computation could exist in some dimension of mind (appropriately defined), leading to a form of idealism (Kastrup, 2018). The metaphysical possibilities are endless! But to keep focused on the direct/indirect issue, I neglect further philosophical debates concerning 'what reality is made of', and simply speak as if it is 'made of matter' - whatever that is! (And terms like 'matter-based reality' here are simply convenient short-hand for the physicalists' 'matter/energy/spacetime-based reality'.) Just for expository ease, when speaking about the indirect school I will sometimes use a specific hypothesis for the basis of conscious experience, namely strong emergence (Chalmers, 2008), viewed as a form of non-reductive physicalism (Stoljar, 2021) [specifically the form in which a complete account of natural phenomena can be given by adding qualia-valued symbols (Rosseinsky, 2021a) to physical theory]. [An explicit example of a 'qualia-valued symbol' - namely '〈tree $\rangle$ ' - will be developed in section 3. Arguments here don't depend on any specifics in the illustrative choice of strong emergence, i.e., reasoning holds for every basically-coherent hypothesis (Rosseinsky, 2021a, 2021b),]

As we'll see, bypassing certain sets of metaphysical issues (physicalism, idealism, computationalism ... identity-theory, emergentism, property-dualism ...) does not leave 'nothing to be said'. Instead, it allows us to focus on the crucial and resolvable issue for phenomenal consciousness (Rosseinsky, 2021a, 2021b): what are the dynamical, information-transfer, relationships between brain and conscious experience? Surprisingly, this question will be the key to creating a contradiction-free candidate in the direct/indirect arena.

In everyday life, it seems that the dominant (near-universal!) habitual tendency is to interpret the contents of visual conscious experience as if identical to matter-objects. That is, naïve realism seems to be the default folk-theory (Blackburn, 1996) (although we should distinguish between heuristic 'as if' models, and deeper 'when pressed' beliefs). But in behavioral science, the default position is usually some version of indirect realism. [For example, consider the extensive and ongoing debates concerning the utility of report-of-experience, including: (Dennett, 1991) vs. (Chalmers, 1996); (Cohen \& Dennett, 2011) vs. (Fahrenfort \& Lamme, 2012); (Rosseinsky, 2015) vs. (Gamez, 2014); and, (Doerig et al., 2019) vs. (Tsuchiya, Andrillon \& Haun, 2019). The specific forms of these debates simply do not arise if we assume all observers are 'seeing the tree directly'. However, as I will argue, the current central thesis in mainstream behavioral science just does not work as science - which, I suggest, might indirectly explain some of the interest in the direct stance!] 
Put simply, who is right? The direct realists, who say that the distal object and the consciouslyexperienced entity are (more-or-less) identical? Or the indirect school, who assert that conscious experience of an object depends on the properties of the distal object, as translated by the brain - but is distinct from it?

\section{$2 \quad$ Rules of Engagement, Reasons for Engagement!}

How are we to answer this question? What methods of comparative evaluation are valid? What can we - the presumed protagonists in the debate, and the fascinated audience! - take as shared prior data or 'established facts'? I generally find that these questions are not separable from the why question: why are we trying to find out?

Say you and I start from totally-inflexible and mutually-contradictory priors. If we then try to make sense of something controversial, in all likelihood we will end up with conflicting answers that make total sense to each of us personally. But little will have been achieved. To first order: if Alice is strongly committed to direct realism and Bob to indirect realism, there is no way for either to 'prove' to the other that they are wrong. Arguments will come down to 'whose construction is simpler'. And simplicity is frequently in the eye of the beholder.

My own preferred approach to the direct/indirect question is as follows. First, I am a scientist. Therefore, I roughly take the data, theories, and overall method of science as a tentative hypothesis. (As we will see, tentativeness must be real and deep, in this area.) Then, I try to frame both direct and indirect interpretations in the resulting scientific frame. This illuminates the pros and cons of the two camps, allowing readers who share the same prior - an interest in science-congruent exploration - to make their own decisions. (Of course, I also take the liberty of sharing my own view!) Remarkably, the process of exploring the direct/indirect controversy will also illuminate severe problems for certain formulations of science itself. To make a maximal advance on the direct/indirect question will then require the willingness to examine, and even rewrite certain foundational tenets of the currentscientific canon - if shown to be necessary and appropriate, on scientific grounds. (And this is where 'deep tentativeness' comes in: we assumed that foundational canon to begin with. But rationality will demand its radical re-examination.)

Now, for many who start from a science-invested prior, there's apparently no need to investigate deeply: it's obvious (allegedly!) that conscious experience must be based on brain-activity, which means that direct realism must be wrong. (I briefly rehearse some of the reasons for these 'musts' in section 5.) Why then, as a scientist, am I writing this Chapter?! Simply put, current practice in the mainstream science of consciousness [e.g. (Melloni et al., 2021)] is fatally flawed, and the 
direct/indirect debate shines light on profound contradictions in that practice. Thus, although I end up advocating strongly for the indirect view, I also argue that the current mainstream-science version of indirectness is just as ad hoc and unattractive as the current philosophical version of directness. What's needed is a fundamental innovation in science - justified by yet-to-be-collected experimental data, interpreted in a rigorous manner - which allows for coherent statement of the indirect position.

So far, I've implicitly addressed the 'how to decide' issue, by declaring an affiliation with science, and sketching an associated style-of-approach. These beginnings also give a partial answer to the 'why try to decide' question: 'because we want a scientific explanation' (or enjoy looking for one!). At the end of this Chapter, I'll point to another 'why': because we want a science that works in the context of society-as-a-whole. As we proceed, I'll link the fatal flaws in current consciousness science (and their remedy) with fatal flaws in the current foundations of science-as-a-whole (and their remedy). In closing, section 15 will briefly link those fundamental (and fixable) issues for the overall enterprise of science to current societal conflicts between science and postmodernism [in particular, the 'post-Truth' movement (McIntyre, 2018)]. Remarkably, then, it will turn out that the direct/indirect debate isn't merely an esoteric debate at the frontiers of science and philosophy. Getting it straight has traction on some of the most dramatic issues presently facing human society. The ultimate why? Because we want a society that works!

\section{$3 \quad$ New Symbol-based Notation: Conscious Experience Distinguished from Object}

In mathematical logic, it's often possible to prove anything if we start from contradictory axioms. (For example, adding the axiom ' $0=1$ ' to a previously-consistent arithmetic, it's possible to prove any number is equal to any other number!) An astonishing feature in the present mainstream practice of consciousness science is the way in which extremely-subtle contradictions are typically introduced before scientific papers have even begun! As I will show in outline in this Chapter, the joint presumption of a dynamically-conventional setting together with the mere statement of an allegedlyscientific term referring to any aspect of conscious experience is already a contradiction. [A precise and rigorous - non-'sketch'! - a demonstration of this and related points can be found elsewhere (Rosseinsky, 2021a). 'Dynamic-conventionality' is explained in more detail in section 8, but for the moment it can be taken as 'restriction to the kinds of dynamical transfer-of-information that the current structure of fundamental physics allows'. Notably, the pivotal issue will be whether information concerning degrees-of-freedom 'inside' a strongly-emergent property can be transmitted by systems that conform to the limitations of current physical law. This will turn out to be more or less synonymous to the question, 'can dynamically-conventional matter-brains reliably report on their own 
experience?'. And if, as indirectists must claim, knowledge-of-the-world is mediated by experience, the brain/experience relationship is crucial to our capacity to have and share such knowledge.]

Clearly, the fact of contradiction in mainstream conscious-experiential science is not widely appreciated, certainly not in science itself. [For example, papers about 'consciousness' have made a recent reappearance in the flagship journal Science (Nieder, Wagener \& Rinnert, 2020; Melloni, Mudrik, Pitts et al., 2021).] The contradiction has direct bearing on the direct/indirect debate, because philosophical-directness can't reasonably be criticized on grounds of incoherency by a selfcontradictory scientific-indirectness! More directly still, illustrating the contradictions in current consciousness science begin with the same means as are required for stating and addressing the direct/indirect debate. These 'means' are a formal symbolism that can make a contrastive statement of the two viewpoints involved. Ultimately and constructively, contradictions must be eliminated, in order to create a rational (indirect) candidate that can be science's leading contender for direct/indirect resolution. This is our direction-of-travel, and symbol-enabled logic will be our vehicle's engine.

Before declaring new symbols, let's address the claim that symbolism by itself can't resolve anything: it's just a linguistic convention. Of course, this is true. And yet most scientists would agree that science can't function without appropriately-defined symbols. Having stated the debate, I'll immediately bypass it in favor of demonstrating the utility of new symbolism in this arena. As I'll show, its key benefit will be to bridge the gap between physical sciences that exist centrally as symbol-stated theories, and behavioral-science/philosophical debates that take place mainly in everyday words.

Consider the matter-constituted tree. In physical science, we could in principle list all its fundamental constituents, as an electron here, some quarks there, some more electrons over there (plus gluons, photons, and so on ...). This is more than a conceptual appeal: this list is (or more precisely, points to) what we mean by 'matter-constituted tree'. Without explaining all the notation (and glossing over all sorts of quantum-field theoretical nuance), this list might look like (a very long version of!):

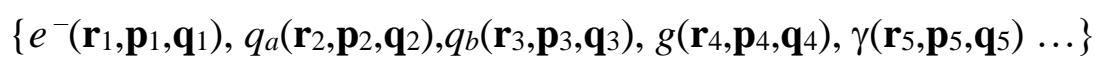

Perhaps more familiar would be a list that states the physical configuration of the tree in terms of chemical elements, $H$ for hydrogen, $C$ for carbon and so on (although some physical detail is already lost at this description-level):

$$
\left\{H\left(\mathbf{r}_{6}, \mathbf{p}_{6}, \mathbf{s}_{6}\right), H\left(\mathbf{r}_{6}, \mathbf{p}_{6}, \mathbf{s}_{6}\right), C\left(\mathbf{r}_{6}, \mathbf{p}_{6}, \mathbf{S}_{6}\right), N\left(\mathbf{r}_{6}, \mathbf{p}_{6}, \mathbf{s}_{6}\right) \ldots\right\}
$$


[Briefly - because these details don't carry forward - in (1) and (2), $\mathbf{r}_{i}$ and $\mathbf{p}_{j}$ are illustrative variables labelling location and momentum, respectively, and $\mathbf{q}_{k}$ and $\mathbf{s}_{l}$ are illustrative variables labelling all other non-r/p properties, such as spin or color state, or 'internal' configuration of an atom.]

Noe let's introduce the new symbol tree to stand for the detailed elementary-particle list given in (1). ('tree' is italicized specifically to distinguish it from everyday 'tree', in the same way that scientific symbols are usually italicized - for example, $H$ for hydrogen. In actual scientific practice, of course, object-1 or $o_{1}$ would be better symbol choices than tree, precisely because 'tree' and 'tree' are so close. For introductory illustrative purposes, however, tree is perhaps more directly accessible to those readers with an aversion to technical symbols.)

To avoid technical, linguistic, debates, when I say 'stand for', I mean that the long-list (1) and the symbol tree are everywhere interchangeable, in science. Both tree and its equivalent list-(1) point to or label the matter-constituents of the tree. And this is one thing we want, for direct/indirect debates: a labelling of the matter-tree. (Declaring symbols like 'tree' isn't typical in physical science, because there it's contextually assumed that the everyday word 'tree' means exactly the same as 'tree'. Thus, adding a formal tree symbol doesn't really give us anything in non-conscious-experiential science. Nevertheless, these kinds of additions are - or should be! - both clear and uncontroversial.)

For direct/indirect debates, we're still lacking an analogous symbolism for 'conscious experience of the tree'. So, let's just invent one! Let's label that exact (visual) experience with this symbolism:

$\langle$ tree $\rangle$

This is where the 'tree' symbol gains its primary value: we can use it do declare $\langle$ tree $\rangle$, as a contrast (Rosseinsky, 2022a). (Angle-brackets in this convention have nothing to do with their usage elsewhere in mathematics or physics. Notably they have nothing to do with bra-ket notation in quantum theory.)

More generally, any matter-object labelled by object gives rise (when witnessed!) to a corresponding conscious-experience labelled by $\langle$ object $\rangle$. It may seem like we have gained very little (apart perhaps from some meager abbreviation-value!). But looks can be deceiving, as I'll show shortly. [Certainly, there's some more technical work to do to ground this symbolism. For example, the general theory eventually wants symbols for the 'atomic' components of conscious experience, across all sensory modalities, not just for the gross declaration of visual-only experience, with all perceptual details wrapped into one symbol. But those extensions are relatively straightforward (Rosseinsky, 2021a).]

\section{Direct Realism, Symbolically Stated}


Rather than defending (3)'s new symbolism against philosophical (and scientific!) critique, I'll just move directly to demonstrating its utility. [Criticisms and responses can be found in (Rosseinsky, 2021a).] Loosely (as the brevity of a single Chapter demands), let's add the further symbolism ' $x \equiv_{\mathrm{P}} y$ ' to mean 'the apparently-two natural features labelled or pointed to by $x$ and $y$ are in fact one and the same thing'. That is, ' $\equiv$ P' stands for physical identity (once we unwrap the fact that scientific symbols are pointers-to features-of-reality).

Then the direct realist view, simply (but approximately) put, is

$$
\text { tree } \equiv_{\mathrm{P}}\langle\text { tree }\rangle
$$

And Eq. 4's symbol-based statement can now be used to illustrate those elementary physics-based arguments which sometimes lead science-based investigators to dismiss the direct view out-of-hand!

\section{$5 \quad$ Simple Physics-based Arguments Against Direct Realism}

\subsection{Energy Conservation}

Illusions are one well-known battleground for the direct/indirect debate (Austin, 1962; Ayer, 1940, Moore, 1953, Putnam, 1999, Russell, 1912). Here I'll give a different spin on this well-worn conversation. Look at a tree. Cross your eyes. If you're at the right distance, you'll experience two semi-overlapping instances of $\langle$ tree $\rangle$. Let's call these $\langle\text { tree }\rangle_{1}$ and $\langle\text { tree }\rangle_{2}$. However, if Eq. 4's physicalidentity of tree and $\langle$ tree $\rangle$ holds, that apparently means there must also be two matter-trees: tree $_{1}$ and $t_{r e e_{2}}$ ! So merely by flexing my face and neck muscles a bit, I've violated the conservation of massenergy, creating a new tree out of nothing! (For a case with two non-overlapping experientialrepresentations, use an arm's-length viewing of your own thumb.)

This experiment seems to imply that direct realism is inconsistent with one of the most fundamental and basic laws of physics. Now, the direct camp can of course patch up their theory. (Because Eq. 4 is a strawman-caricature of directism, there's genuinely some room to do patching!)

For example, directists may say 'We don't really mean tree $\equiv_{\mathrm{P}}\langle$ tree $\rangle$, in the way you're interpreting it'. One possibility is something like $\chi[$ tree $] \equiv_{\mathrm{P}}\langle$ tree $\rangle$, where $\chi[\ldots]$ means 'direct contact of consciousness with ...' Fair enough - and this is a better statement of the directist position than Eq. 4. But then why does flexing some small muscles change the action of $\chi$ ? The only semi-consistent way to understand directist- $\chi$ seems to be as a sort of consciousness-spotlight that's welded to my head (so that as the head moves, what's illuminated changes). To explain the capacity to focus near-and-far we need a model of two beams-of- $\chi$ coming out of the eyes. In fact, the focus of these $\chi$-beams needs 
to be precisely attuned with flexion and relaxation of the lenses in the eyes. It's all getting very convoluted. But that's not definitive: multi-factorial simplicity is in the eye of the beholder! Where all this seems to end up is that directists must posit some sort of non-physical light (or 'consciousness radiation' of some kind), coming out of the eyes and illuminating the physical scene: it's hard to see how to explain focus-in-vision and splitting-in-eye-crossing in any other way. (This does lead to a host of further problems. Why does turning out the physical light stop the consciousness-radiation from functioning? Why do certain brain-injuries affect vision? And so on.)

The primary point here is to illustrate why those invested in physical science often immediately reject directism: e.g., there was that term 'non-physical' in there! Secondarily, I want to illustrate the multifactorial issue. Directists may be (consciously or unconsciously) in contact with knowledge-related problems in dynamically-conventional indirectism (which we'll come on to, shortly). Thus, they may prefer to lose knowledge about how consciousness works - by proposing non-physical mechanisms that seem impossible to investigate - than to lose knowledge about the world itself. (In their view, indirectism entails the latter loss.) Conversely, consider indirectists who deny or who are unaware of dynamically-conventional knowledge-problems for their view. Of course, they'll see directism as simply a cost ('non-physical'!) without a benefit. This can lead to out-of-hand dismissal. The goal of this Chapter is to see if there is a middle ground between these two flavors of directism and indirectism.

\subsection{Experiential Geometry}

One fascinating extension of $\langle\ldots\rangle$-symbolism (Rosseinsky, 2022c) formalizes the undeniable fact that experienced representations of objects occur at distinct relative locations in conscious-experiential space (Smythies, 2003; Wagner, 2006). The relationship between these conscious-experiential locations, and locations in conventional spacetime, is then a matter of great and productive theoretical interest (Rosseinsky, 2022c). For now, let's adopt a notation $\mathbf{r}_{1}, \mathbf{r}_{2}, \ldots$ etc. for coordinates on conventional spacetime, and $\rho_{1}, \rho_{2}, \ldots$ for conscious-experiential coordinates. For example, glossing technical details, we could label the location of tree by $\mathbf{r}_{1}$ and the location of $\langle$ tree $\rangle$ by $\rho_{1}$. [This notation doesn't inherently assert any particular relationship between $\mathbf{r}_{1}$ and $\boldsymbol{\rho}_{1}$, for example, ' $\mathbf{r}_{1} / \boldsymbol{\rho}_{1}$ are just two different labels for the "same place",, That is indeed one option. But there are many others. And every single one, including ' $\mathbf{r}_{1} \equiv_{\mathrm{P}} \boldsymbol{\rho}_{1}$ ' raises troubling questions for conventional, local, scientific theory (Rosseinsky, 2022c).]

Turning to the direct/indirect debate, consider the fact that there appear to be two very distinct geometries for the two spaces indexed by $\mathbf{r}$ and $\rho$ coordinates. Physical measurements show that $\mathbf{r}$ space is locally flat [i.e. Euclidean (Vardanyan, Trotta \& Silk, 2009)]. But $\rho$-space is self-evidently 
non-Euclidean: parallel lines converge (French, 1987). This is extremely problematic for an indirect view. If tree $\equiv_{\mathrm{P}}\langle$ tree $\rangle$, then the same thing holds for the spatial coordinates: $\mathbf{r}_{1} \equiv_{\mathrm{P}} \rho_{1}, \mathbf{r} / \rho$ coordinates are just different names for the same locus, and geometry (distance-relationships between coordinatepairs) must be the same on $\mathbf{r}$ - and $\boldsymbol{\rho}$-spaces. Yet this leads to a contradiction: $\mathbf{r}$-geometry (Vardanyan, Trotta \& Silk, 2009) is flat, and $\rho$-geometry (French, 1987; Wagner, 2006) is not! [Now, for a balanced view, the indirectist also has a severe $\mathbf{r} / \rho$-problem to solve: where does the geometrical structure of $\rho$ space come from? It differs from r-space. And it's nowhere to be found in the brain: there are no known 'Cartesian theaters' (Dennett \& Kinsbourne, 1992) - neuronal arrangements or dynamics that recapitulate or encode subjectively-experienced non-Euclidean geometry. While this can be resolved in an appropriate indirect setting (Rosseinsky, 2022b), it's unfortunately beyond present scope.]

\subsection{Speech Acts: How Does Conscious-Experiential Information 'Get Into’ Brain-Dynamics?}

Suppose I say, 'I see the tree - its greenness, the way those twigs intertwine in space ...'. What is directist-Diane's explanation of this speech act? Suppose Diane believes my vocal cords are governed by my brain. And suppose Diane also holds that $\langle$ tree $\rangle$ does play some actual role in generating the speech act. (I am tempted to write 'causing' the speech act, but I know better than to dare such usage for a mixed scientific/philosophical readership!) Then Diane is in a very difficult position (at least if she also believes in local information transmission). For the experienced/matter-tree is at a distance from me. There's no apparent mechanism - without proposing a whole new layer of reality - for the greenness and twiggy-ness and so on to 'get into' the brain-activity that generates the speech act 'I see a green, twiggy tree'. (Of course, Diane can propose a non-physical mind that communicates with the physical brain. Personally, I object to the use of non-physical/physical in this way - although I have used it above! - because scientists usually take 'physical' to mean 'real' or 'actually existent'. Thus, I would rather describe this proposal as 'mind not instantiated in physically-conventional matter'. However worded, this doesn't usually seem to be the directists' want, though: they want the mind to still live in the skull, so to speak, and witness tree illuminated by $C$-light. Then there still seems to be the problem of communication between skull-bound brain, which generates speech acts, and distal $\langle$ tree $\rangle \equiv \mathrm{P} \chi[$ tree $]$.

Directists could try to say ' $\langle$ tree $\rangle \equiv_{\mathrm{P}} \chi[$ tree $]$ doesn't rule out conventional scientific routes, in which brain-activity is influenced by the physical environment, and so encodes it'. They could say that this conventional route generates the speech act, whereas the experience happens by a different channel via $\langle$ tree $\rangle \equiv_{\mathrm{P}} \chi[$ tree $]$. But this fatally undermines something that directists are often trying to do: to assert that we have direct knowledge of reality, and can speak from that direct knowledge! 
Rather than engage with all the previous to-and-fro between direct and (dynamically-conventional) indirect camps, my approach here is to demonstrate what I believe to be fatal flaws in both, as currently framed - and then offer a third, functional, alternative. Thus, rather than getting into historical weeds, I seek to construct a new framework, and let the open-minded reader apply it for herself, to see how (and if!) entanglements around this topic can be unknotted.

However, any new framework must address a consistent historic concern of the direct school: if we don't see tree, but only create a sort of three-dimensional, brain-based, movie containing things like $\langle$ tree $\rangle$ - how do we get reliable, useful, knowledge about reality?

The answer is both straightforward and extremely subtle.

The straightforward piece: $\langle$ tree $\rangle$ is to $\langle$ bird $\rangle$ as tree is to bird. We do not get direct knowledge of physical reality itself. But we do get knowledge about structural relational features. And that is enough! (This presumes that mechanisms generating $\{\langle$ tree $\rangle,\langle$ bird $\rangle\}$ from $\{$ tree, bird $\}$ preserve key relational features. And this indeed seems to be the case. For example. although $\mathbf{r}$-space and $\boldsymbol{\rho}$-space have different geometries, there is a smooth map between them. So, co-location is the same thing in both spaces. We can infer r-velocity from $\rho$-velocity, well-enough. And so on.)

The subtle piece: mainstream indirect realism faces exactly the same 'speech act' problems as direct realism does (section 5.3), although they originate in a different way. That is, it is not enough for indirectists to give the answer of the previous paragraph, if at the same time they assert a dynamicallyconventional setting! As we'll see, dynamic-conventionality prevents the knowledge-content of $\{\langle$ tree $\rangle,\langle$ bird $\rangle\}$ getting into speech acts! The knowledge-transfer problem occurs for directists in the physical distance between brain and $\chi[$ tree $]$. But for indirectists it occurs in the dynamical disconnect between information inside a strongly-emergent representation, so to speak, and the dynamical activity of the system which informs that representation (i.e., a brain), and then (allegedly!) reports on it.

\section{Indirect Realism, Symbolically Stated: New Symbolism for Encoding}

The rest of this Chapter will be concerned, then, with giving an indirectist account that can answer the directists' concerns about knowledge of the world - in a non-contradictory way. (Again, the current mainstream practice of consciousness science assumes such an account has already been given. Again, I will show why that mainstream practice is contradictory.) 
To state and explore the indirect position, let's now introduce two further symbolic components. First, write Enc(object) to label the brain's encoding of object. (Here 'object' is a scientific symbol like tree.) There's some work to do to establish precisely what Enc means (Rosseinsky, 2021a). But roughly speaking, Enc(tree) labels those components of brain-activity that can be decoded by an idealized, perfect-information, third-party computational-neuroscientist, who doesn't know what the brain is actually observing. Thus, tree, Enc(tree), and $\langle$ tree $\rangle$ point to the triad of distal object, object-related brain-activity, and conscious experience of object. A key component of the indirect view, then is that

$$
\text { tree } \left.\rightarrow_{e} \text { Enc(tree }\right) \rightarrow_{e}\langle\text { tree }\rangle
$$

where ' $\rightarrow e$ ' stands for ' $e$-causes', drawing on a carefully worked-out ' $e$-casual' conception of dynamic relationship that should be acceptable to both scientists and philosophers (Gamez, 2014). Of course, a complete indirectist account requires more than that, for example clearly asserting that there isn't anything like $\chi[$ tree $] \equiv_{\mathrm{P}}\langle$ tree $\rangle$ going on, as well. For definiteness, in simple language, the rest of this Chapter tentatively presumes that tree and $\langle$ tree $\rangle$ are non-identical, i.e. that indirect realism holds. But that tentative presumption is not the end of the game, because we still need to see (per section 6) if it can be made in a way that preserves knowledge-of-the-world, and is non-contradictory, in and as science.

As I noted in section 3, just declaring assumptions or axioms, without examining the consistency of the resultant, overall, axiom set, can go badly wrong. Here, we now need to examine whether asserting tree $\langle\langle$ tree $\rangle$ non-identity (and discarding the $\chi[$ tree $]$ alternative) leads to contradictions with pre-existing axioms of science. Notably, there is a particular pre-existing assumption, built into the foundations of current mainstream science, which I call dynamic-conventionality. The primary question now becomes: can non-identity (indirectism!) and dynamic-conventionality co-exist without contradiction, and give us something that looks recognisably like science-as-we-know-it?

\section{Dynamically-Conventional Physics Denies Scientifically-Reliable Report of Experience}

As far as I know, no-one doubts that Enc(tree) can $e$-cause (Gamez, 2014) a speech act of the form 'I am now consciously experiencing a tree'. But we can program quite basic computers to parse data generated by cameras, and make analogous, object-veridical, reports! ('Object-veridical' means when the camera is directed at a tree, the report is 'tree'. When it's directed at a car, the report is 'car'. And so on.) The crucial question concerns the $e$-causal role of $\langle$ tree $\rangle$ in such reports, not that of Enc(tree). [Although I won't pursue this here, Enc(tree) and $\langle$ tree $\rangle$ are two different kinds of 'representation', e.g., per this volume's title. And the Enc/ $/ \ldots\rangle$-distinction is more useful scientifically than the 
philosopher's 'phenomenal/access'-distinction for consciousness (Block, 2005; Block, 2007), because 'accessible' just means 'reportable'. The useful scientific fundamentals are tree, Enc(tree), $\langle$ tree $\rangle$ and report-data (Rosseinsky, 2021a).]

It's not possible to review the entire literature here concerning the report-role of $\langle$ tree $\rangle /$ Enc(tree). [See for example (Wilkes, 1988; Elitzur, 1989; Rudd, 2000; Chalmers, 2013; Phillips, 2018), plus the other adversarially-styled pairings mentioned in section 1. (Rosseinsky, 2021a) discusses these and other contributions.] But roughly speaking, the modern mainstream science of consciousness proceeds along these lines. $\langle$ tree $\rangle$ is taken to be 'identical' in some sense to some subset of Enc(tree). ['Identity' here includes conventional emergence. Leading theories (Melloni et al., 2021) typically posit that identity in this general sense only occurs under the co-presence of other, consciousness-enabling, brainactivity. But notation and reasoning generalize in a straightforward way.] Let's write this subset as $E n c_{\langle\ldots\rangle}$. [For example, part of the 'neural correlates of consciousness' program (Gamez, 2014; Koch, Massimini, Boly et al., 2016) aims to determine exactly which Enc-subset Enc(..) is.] Then because $E n c$ can $e$-cause speech acts, and because $E n c\langle\ldots\rangle$ and $\langle\ldots\rangle$ are taken to be 'identical', we can apparently attribute speech $e$-causality to $\langle\ldots\rangle$. For humans then (as opposed to a hypothetical, non-conscious, machine), 'I am now consciously experiencing a tree' looks like it meaningfully derives from $\langle$ tree $\rangle$. This seems to be a resolution of the knowledge problem, because it suggests we can collectively, intersubjectively, enquire into $\{\langle$ tree $\rangle,\langle$ bird $\rangle\}$ relationships! You can say ' $\langle$ tree $\rangle$ ', and I can say ' $\langle$ bird $\rangle$ ', and the speech acts look like they originate in your actual- $\langle$ tree $\rangle$ and my actual- $\langle$ bird $\rangle$.

However, if all this is set in a dynamically-conventional context, it cannot go through. Informally, 'dynamically conventional' science excludes the influence of conscious experience on matter-states, specifically on the matter-states of the brain that's 'having' those conscious experiences. [See (Rosseinsky, 2021a) for a more precise characterization.] This is the frame of mainstream modern science, if we read it as simply excluding 'mind over (brain) matter'! Of course, mainstream modern consciousness science says that a precise kind of 'influence' is $\mathrm{OK}$, namely (and only!) the $e$-causal consequence of emergent brain-states that both encode tree as Enc(tree) and 'are' $\langle$ tree (as $E n c\langle\ldots\rangle($ tree $))$. So, a bit less informally, 'dynamically-conventional science forbids e.g. a $\langle$ tree $\rangle$-labelled phenomenon any e-causality over and above that attributable to its Enc(..)(tree), brain-activity, substrate'. Here, though, is the entire rub.

For consciousness to 'influence matter' in a way that allows for knowledge, there must be dynamical innovations in brain-activity, over and above the conventional-physics dynamical account, by virtue of the presence or existence of conscious experience. (The kind of 'knowledge' supported by this kind 
of mechanism is brain-instantiated knowledge of experience. This is not the strong form that some directists want. But without it, arguably under physicalism no kinds of conscious knowledge of the world exist.) However, such dynamic innovations are ruled out by dynamic-conventionality. Mainstream consciousness science asserts that, in its theories, there are no such innovations, thus assuring that it can co-exist with the rest of the current, dynamically-conventional, version of science. This is a correct evaluation of its own theories. But mainstream consciousness science is incorrect to then assert that a dynamically-conventional science can support an experimental enquiry into conscious experience! If there are no 'dynamical innovations in brain-activity ... by virtue of the presence or existence of conscious experience' (the dynamically-conventional stance), then no information about any facet of conscious experience can be reliably reported by (or 'via') a brain! There is nothing - dynamically - to originate or ground those reports. So, they are not reliable data in alleged experiments. And they are not reliable data more generally in human attempts to 'know the world'. (More strongly, as section 12 discusses, if our thoughts are brain-generated, the idea that these thoughts are 'about' conscious experience must be delusional, in a dynamically-conventional reality.)

Put bluntly, simple identity or weak-emergence or strong-emergence (or any of a literally-infinite raft of metaphysical theories of conscious experience) could be true. But if they are true in a dynamicallyconventional setting, then no speech acts are meaningfully or scientifically about conscious experience itself. Thus, the directists concerns about knowledge of the world can't be resolved by dynamicallyconventional indirectism.

\section{$9 \quad$ But We Know We Can Report on Our Conscious Experiences!}

One common response to 'there are no reliable speech acts about conscious experience' is: 'This is nonsense! I know I can speak of the greenness of that tree - I'm doing it right now. If I were to doubt that, it would be impossible to speak about anything.' Emphatically: I do not deny the capacity to speak meaningfully and reliably about conscious experience. Logic denies that capacity in a dynamicallyconventional reality. Thus, if we were to incontrovertibly assert the capacity for reliable report, the only logical conclusion would be that our Universe is not dynamically-conventional. But this move typically leads to (somewhat understandable) outrage from scientists, and (somewhat incomprehensible) outrage from (some) philosophers.

Scientists can validly object to reasoning from subjective assertion to dynamic-unconventionality, because dynamic-unconventionality does require some fundamental-style revision to physical theory: physicists appropriately require higher evidence standards than 'it seems so to me', for such revisions! However, there is an experiment that can investigate the dynamic character of our reality (Rosseinsky, 
2021b). Reasoning about speech acts - developed in more detail in what follows - simply points strongly towards that experiment!

Philosophers typically object to dynamic-unconventionality because it allegedly 'violates causal closure'. This is simply a mistake. Saying that there are dynamic innovations in brain activity traceable to the influence of $\langle\ldots\rangle$-phenomena just doesn't violate causal closure. Consider strong-emergence with downward causation. (Again, there are literally an infinity of other conceptions that both contain dynamic-unconventionality and honor causal closure. What they don't honor is causal closure with respect to the current set of fundamental entities and their current interaction rules. But trying to hold those fixed - and obstructing experiments that could revise them - is simply to the incomprehensible assertion that 'fundamental science is finished'!) Another style of philosophical objection is that dynamical-unconventionality is 'giving up' (Dennett, 1991). It is not. It is pointing out that, logically, rationally, we must give up on consciousness science - and in fact on the conventional interpretation of science itself (section 12) - if reality is dynamically-conventional! Hypothesizing and then experimentally-testing dynamic-unconventionality is actually the maximal form of not giving-up, that's consistent with non-contradictory science!

\section{Impossibility of a Dynamically-Conventional Experimental Science of Conscious Experience}

As noted above, the brain/experience relationship and the capacity-for-report have already been hotly and extensively debated. Simply declaring some allegedly-definitive personal views arguably doesn't add anything. But precise symbol-based reasoning can (Rosseinsky, 2021a). So far, I've demonstrated some helpful abbreviation-capacity of tree/〈tree $\rangle$ formalism. Next, I will illustrate the real role I asserted for it in section 3, in '[bridging] the gap between physical sciences ... and behavioralscience/philosophical debates ...'.

Physical science already has an explicit theory of what's needed for a system to be able to make reliable reports on some degree-of-freedom. It uses the well-established Hamiltonian formalism for describing time-evolution of the system (Goldstein, 2001), and uncontroversially requires (Bohm, 1951) the existence of an interaction term of the form

$$
H_{\text {int }}=-g(t) \cdot f(p, l)
$$

Here $g$ and $f$ are mathematical functions whose precise character we can gloss over (for a first-pass). Crucially, though, $f$ describes a physical interaction between some property $p$ of the report-system, and the quantity $l$ that the system is supposed to be reporting on. 
Because we now have 〈...〉-symbolism that's embedded in physical theory, we can apply this Hamiltonian-based analysis from mathematical physics to the contentious question of report-ofconscious-experience. Notably, if $l_{\langle\ldots\rangle}$ indexes distinct characteristics 'inside' conscious experience, to report on those characteristics we would need the existence of terms like

$$
H_{\mathrm{int}}=-g(t) \cdot f\left(p, l_{\ldots \ldots}\right)
$$

But this is exactly what 'dynamical conventionality' forbids!

Reasonably, you may still feel that symbolism hasn't added much yet to debates: Eq. 7 can be read at first as just another statement of what's needed for reliable report, and the dynamically-conventional indirectist can try to claim that Eq. 7's $l_{\langle\ldots\rangle}$-coupling does exist, because (unarguably) Enc-coupling does and (metaphysically-hypothetically) $\langle$ tree $\rangle$ can be viewed as 'identical' to Enc $\langle\ldots\rangle($ tree $)$. Here is where symbolism does add something. Accepting the indirectists' premises (Enc-coupling and identity), it is possible to show that these are simply not enough to ground an experimental science of conscious experience. A second read of Eq. 7 emphasizes the key point: it's not enough for there to be some generic coupling. For an experimental science of conscious experience, different l-values (corresponding to different theoretical hypotheses) must be able to generate different outputs (speech acts about conscious experience). [In philosophically-technical terms, the brain must be able to make reports on 'phenomenal judgements' (Chalmers, 1996; Rudd, 2000).] And that is forbidden by dynamic-conventionality.

For an introductory exposition of this point, see (Rosseinsky, 2015) as a commentary on (Gamez, 2014). [(Gamez, 2014) is a precise and excellent account of the current neural correlates of consciousness, or 'NCC' program. (Rosseinsky, 2015) exemplifies the point that every statement of that program implicitly assumes the existence-in-reality of Eq.7-equivalent couplings, thus contradicting the dynamic-conventionality assumption that every mainstream consciousness-science program also adopts. The NCC program isn't inherently contradictory or impossible. But dynamicallyconventional NCC research is!]

(Rosseinsky, 2021a) gives a complete analysis of the role of $l_{\langle\ldots\rangle}$-resolution in a reliable science of conscious experience, and how it can be addressed. $\left(l_{<\ldots .}\right)^{\text {'‘ }}$ resolution' is the capacity to make different reports when different $l_{\langle\ldots\rangle}$-indexed natural-states occur.) To ground present discussions, a brief sketch of that demonstration follows.

Consider an early question in the modern renaissance of conscious-experiential science: 'does activity in early visual-processing brain-area $V 1$ play some direct part in conscious experience?' (Crick \& 
Koch, 1995). [The basic neurophysiological fact required for this discussion is that human-VI encodes the visual environment at the finest level of cortical detail. Later areas aggregate information, and thus contain coarser encodings. Figure 1 in (Rosseinsky, 2015) illustrates differing detail-levels.]

Consider two hypothetical theories. In theory-1, VI-activity does play a role, and conscious visual experience is finely-grained to a level we will index by the $l_{\langle\ldots\rangle}$-value $l_{\langle\ldots\rangle, 1}$. In theory-2, $V 1$-activity doesn't play a role, and we label all coarser experiences $l_{\langle\ldots\rangle, 2 .}$. (We have now concretized the generic $l$ in Eqs. 6 and 7, and devoted it to indexing different granularities in visual encoding and experience. The precise numeric values we give to various $l_{a}$ instances here does not matter. The fact that $l_{\langle\ldots\rangle, 1}$ and $l_{\langle\ldots\rangle, 2}$ correspond to two different well-defined theories is what we need. For example, a subject reports 'I consciously experience $V l$-congruent detail' is the assertion $l=l_{\langle\ldots\rangle, 1}$.) To perform theory-1/theory2 arbitration based on experimental data - i.e. to do science - experimental subjects must be able to make $l_{\langle\ldots\rangle, 1} / l_{\langle\ldots\rangle, 2}$-resolving reports. And this is what dynamic-conventionality forbids.

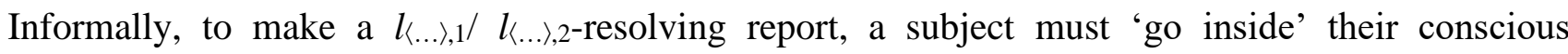
experience, compare what's in there to $V 1 /$ later-than- $V 1$ benchmarks, and bring the results back into speech. 'In there' means 'in conscious experience': to do this, there must be the capacity for information to transfer from within conscious experience, into brain-dynamics. The dynamicallyconventional indirectists' assumptions (Enc-coupling and identity) are enough to give a general account of the existence of conscious experience. (Although, so are many other approaches!). But, because they deny required information transfer, they cannot lead to an experimental science of conscious experience, under the dynamically-conventional assumption. (That is, on the presumption that science still requires a rational, contradiction-free, account of how its measuring devices function!) Somewhat aware of these issues, dynamically-conventional mainstreamers have assembled a patchwork of responses [e.g. (Tuschiya et al, 2019)]. But these aren't successful either individually, or as a collection (Kleiner \& Hoel, 2021) (Rosseinsky, 2021a). They can't be, because of the Eq.7/dynamic-conventionality mutual-contradiction.

\section{From Experimental Report, to States of Consciousness, to Definitions of Consciousness!}

Whether $V 1$ plays a part in conscious experience, and the corresponding question of the precise level of granularity in experience, may seem to be marginal issues. (Unfortunately, they can get confused with the resolution level of the encoding brain, thus muddying the waters a little.) One response is: 'I can doubt my ability to report on detail-level. But I can't doubt my ability to report on large-scale features of experience, or on the existence of experience itself.' However, theory-1/theory-2arbitration and its required $l_{\langle\ldots\rangle, 1} / l_{\langle\ldots\rangle, 2}$-resolution are not marginal, technical, issues. The problems 
they raise generalize immediately to report on each and every aspect of conscious experience, including whether we can meaningfully speak of conscious experience at all, in a dynamicallyconventional world! [For careful generalization, see (Rosseinsky, 2021b). Interestingly, for scholars of the field as a whole, Dennett's controversial approach (Dennett, 1991) can be understood as the entirely rational consequence of fixed pre-commitment to dynamic-conventionality. In contrast, the entire mainstream e.g. NCC field can be seen as an irrational consequence of the same precommitment, if we view contradiction as a form of irrationality. Either way, instead of an unscientific/illogical fixed pre-commitment to dynamic conventionality, what we need is experimental investigation of reality's actual dynamic character!]

If an experimental-subject can't talk meaningfully or reliably in-experiment about consciousexperiential features, the scientist-experimenter can't talk about conscious experience writ large in scientific discourse, either (Wilkes, 1989; Dennett, 1991; Rosseinsky, 2021b). For example, papers discussing how we are going to develop a science of conscious experience are gibberish! But doesn't that make this Chapter gibberish? Because, unlike current mainstream scientific discourse, I don't make an inflexible pre-commitment to dynamical conventionality, that charge doesn't apply here. That is, the 'can't and 'gibberish' attributions in this paragraph's two opening sentences presume a dynamically-conventional context, which is not the general presumption in my approach. (But consider section 13's experiments investigating the dynamical character of reality. If these consistently pointed to a dynamically-conventional Universe, then that would be scientific evidence in favour of a gibberattribution to this Chapter. Thus, as a scientist, I hold this discussion as 'contingently non-gibberish', pending the outcome of putative future experiments!)

Interestingly for historians of the modern field, the issue of non-reportability (in fact, unspeakability!) of conscious experience was highlighted at an early point - and then apparently discarded. Chalmers' seminal The Conscious Mind (Chalmers, 1996) goes through an informal analysis of "phenomenal judgement' under a form of causal closure that's a version of dynamical-conventionality. This analysis investigates whether and how we can be sure of our own report of conscious experience. [See Figure 13 in (Rosseinsky, 2021b), for a detailed exploration of Chalmer's approach.] Correctly (given assumptions), Chalmers' analysis concludes that the brain cannot verify its own experiential reports, and suggests instead a mental 'report-to-self', verifying conscious-experience-of-report matches with conscious-experience-that's-reported. However, this ingenious solution does not qualify as causallyclosed: minimally, the computational process that compares experience-of-report with experiencethat's-reported can't have a basis in matter. This doesn't mean that the analysis is wrong - it means it can't be used as a justification for the soundness of experiments in a dynamically-conventional reality. 
(Even if we give the computational comparison-process a 'pass' on having no basis in matter, its output - 'does match' or 'doesn't match' - can't ever be spoken, under Chalmers assumptions, because that would couple matter directly to a result causally downstream from a non-matter process. Chalmerian causal closure forbids that.) Nevertheless, it seems self-verification of report has been taken as a sound basis for experimental science. [Other explicit 'bridging' principles have been proposed (Chalmers, 2013; Gamez, 2014). Because a reliable meter of conscious experience must be $l_{\langle\ldots\rangle}$-resolving, every such principle - if capable of grounding experimental science - must assert that Eq. 7 holds in the human brain. That is, every functional bridging principle must assert dynamic-unconventionality, directly contradicting the dynamically-conventional setting that mainstream consciousness science also assumes. In this sense, mainstream, dynamically-conventional, consciousness science is founded on ' $0=1$ '! ' $0=1$ ' in arithmetic systems means arithmeticians can prove any result they want; perhaps analogously, axiomatic contradiction in mainstream consciousness science may explain why experiments seem to show whatever the experimenters are looking for (Yaron, Mudrik \& Pitts, 2021):]

\section{Physics, Revisited: Dynamically-Conventional Science Can't Be (Conventionally) Reliable}

But the implications of a dynamically-conventional assumption (or reality, if that's what we live in!) don't stop with consciousness science. In fact, followed through to science-as-a-whole (Rosseinsky, 2021c), they show a form of ' $0=1$ ' lurking there, too!

If reality is dynamically-conventional, we can't rely on any use of any aspect of conscious experience in our thought processes. We may reason to ourselves that 'I know that thermometer read 98.4F, because I can recall the consciously-experienced scene where 9-8-4 was the digital reading'. However, no component of conscious experience can influence brain-activity under dynamic-conventionality. So, such self-reassurances must be delusions. And this point applies to scientists' self-reassurances about the collection of experimental data, too, not just to my everyday temperature readings! Moreover, the issue transfers to every other aspect of science that's (allegedly) conducted in conscious experience. Say I'm reviewing a paper, or even my own theoretical musings. The idea that I can reliably 'see' either correctness or falsity, in a conscious-experiential sense, must also be a delusion. (This follows from the view that ' $\mathrm{I}$ ' is really another name for the stream of brain-generated thoughts.) The best that can be happening is that the brain is recalling the thermometer reading accurately, or assessing theories accurately, and simultaneously projecting a movie of that activity into conscious experience. But we can't rely on that movie to assure us of accuracy, because any alleged use of the movie is itself (dynamically-conventional) impossible! It's possible that science is working well, and indeed the success of technology and so on is almost incontrovertible evidence that it is, in those areas. 
Yet it is not working for the reasons we think, and there's every reason to believe that subtle problems would persist unrecognised. (For example, in a dynamically-conventional reality, perhaps a form of consciousness science would arise that is contradictory, but this would go unchallenged, because the basis of scientific process is dysfunctional!)

If reality is dynamically-unconventional, then the foundations of science are currently incorrect (because they assert conventionality!). If unconventionality is of a sufficiently-strong kind (e.g. if all degrees-of-freedom in conscious experience do couple reportably with brain-activity), all the uses of conscious experience in science may be valid. (That is, in this case, they are not provably invalid. They may still suffer from other e.g. cognitive flaws.) Nevertheless, in this case, a profound and vital aspect of reality is being overlooked by an untested assumption in the foundations of science. (Untested assumptions are not wrong, in and of themselves. But if they are testable, they should be tested!)

Put simply, this analysis places dynamically-conventional science in an impossible position. (And this impossibility may be one reason for the persistence of direct realism, because indirectism and mainstream science are more-or-less synonymous, at least in the sense that mainstream behavioral science's consideration of conscious experience is indirectist.) Either it's correct in its assertion that reality is dynamically-conventional, in which case its own account of its own functioning is incorrect, and there's no guarantee that damaging flaws (if they exist) will be corrected. Or it's incorrect in asserting dynamic conventionality, in which case it is just wrong!

Arguably, the best outcome is that current science is simply wrong on this point - because mistakes can often simply be corrected! Fortunately, the actual dynamical character of reality need not be a matter of debate or conjecture: it can be established experimentally, at least in principle.

\section{Is Reality Dynamically Conventional? An Experimental Question!}

Experimental tests for dynamically-conventional $v s$ unconventional reality are conceptually very straightforward (albeit likely extremely challenging, in practice) (Rosseinsky, 2021b). If reality is dynamically-unconventional, systems making reliable-report on conscious experience must show dynamical innovations, as compared to a dynamically-conventional model. Simply, in such a case, complete recordings of actual brain-dynamics must diverge from a complete simulation based on dynamically-conventional physics. [Of course, completeness is not achievable in either aspect of an in-practice comparison of actual-vs.-simulated brain-dynamics. But completeness is rarely the objective in experimental science: more typically, we seek enoughness. Detailed discussions of technical challenges and feasibility for an actual-vs-simulated experimental-program can be found in (Rosseinsky, 2021b).] 
An apparent reluctance on the part of both scientists and philosophers to even discuss actual-vs.simulated comparisons is somewhat curious. Certainly, such an experimental program deserves robust evaluation, before committing what may well be significant funds. It is hard to avoid the sense that parties invested in quite different theoretical approaches share some deep resistance to actually finding out the fact-of-the-matter, concerning reality's dynamical character. Perhaps we are just too conditioned to some version of materialist conventionality to even look on perfectly-reasonable alternatives. Perhaps the default to everyday directism (Blackburn, 1996), no matter what our deeper insights may be, is a significant obstacle to experimental progress on dynamical character. For these experiments - if they support dynamic-unconventionality - offer significant support to the indirect view.

\section{Experimental Traction on the Direct/Indirect Debate?!}

Whatever the prospects for actually embarking on actual-vs.-simulated analyses, they do seem to offer the only visible path to laying the direct/indirect debate finally to rest. We've seen that the two horses in the race are dynamically-indeterminate directism, and dynamically-conventional indirectism. The first seems to preserve the possibility of knowledge, at the cost of science (particularly, it seems, at the cost of scientific knowledge about how we have knowledge!). The second tries to establish a basis for both knowledge and science, but ends up leading to equally severe problems for knowledge in science.

If preserving some form of science isn't an issue for you, it's a matter of personal preference which basket of contradictions and deficits you prefer - the directist or the dynamically-conventional indirectist. A large part of my evaluation-metric for the direct/indirect debate concerns establishing a reliable, non-contradictory place for science, if it's possible to do this in a reliable, non-contradictory way! This place only exists if reality is strongly dynamically-unconventional (Rosseinsky, 2021c). The only way we can see in science that this place exists (if it does!), is to successfully execute an actual$v s .-$ simulated experimental program (Rosseinsky, 2021b). Thus, the direct/indirect debate can in principle be resolved by experiment, but not in the sense that data decisively supports one position over the other. Rather, signals confirming a dynamically-unconventional reality allow us to create a contradiction-free, science-inclusive, indirect position, which allows for reliable (if indirect!) knowledge of the world.

Naturally, directists are still free to insist that $\chi[$ tree $]$ plays a role, and neither the arguments above nor putative future data could disprove that assertion. (For example, consider Diane's 'mind not instantiated in physically-conventional matter'. If this is a 'mind that communicates with the physical brain', it must hypothesize dynamically-unconventional activity of exactly the kind that actual-vs.- 
simulated experiments could uncover.) Nevertheless, it's hard to see why one would want to assert that $\chi[$ tree $]$ is the mechanism of conscious experience, if we can have knowledge, science, and nocontradiction, under the dynamically-unconventional indirect view.

\section{From the Foundations of Science to the Foundations of Society}

While all of this is (I hope!) novel and interesting to object/representation field-specialists, the title of this Chapter asserts a 'remarkable relevance' of the direct/indirect debate to both science and society. Science-relevance of a kind has been established. Concerning broader relevance, it may still look like all I've achieved so far is to connect one esoteric frontier (the direct/indirect issue in philosophy of mind and consciousness science) with another (the role and reliability of appeals to conscious experience, in the process of science-as-a-whole).

Anyone who's been paying any attention to the sociopolitical domain over the last ten years can't have failed to notice the mounting attacks on science, from various kinds of postmodern viewpoints (Baldwin, 2020; Latour, 2010; Sokal \& Bricmont, 1999). Of course, there's a lot more involved in recent attacks on science than a postmodern intellectual critique. [Briefly, we must distinguish an extremist 'post-Truth' version of postmodernism (McIntyre, 2018), attribute the most destructive attacks to this sub-school, and point out that mainstream postmodern advocates do not necessarily subscribe to or support the post-Truth stance. Post-Truth plausibly involves a subversion of wellintentioned intellectual discourse, to serve personal agendas.] Nevertheless, unexamined, unrecognized, and unresolved flaws in the foundations of science make it vulnerable to postmodern attacks. Put differently, if reality is dynamically-conventional, postmodernists are right, in a way: science may not be a completely social/subjective construction (Barnes, Bloor \& Henry, 1996), as they sometimes assert, but its own account of how it isn't is profoundly flawed. And if reality is dynamically-unconventional, postmodernists are again right, in a way: science's definitive proclamation of a meaninglessly mechanical-or-random reality (Weinberg, 1977) is mistaken. (Dynamic-unconventionality need not ultimately establish meaning, authentic free will, or non-random creativity. But it is definitely a potent vulnerability in the otherwise impregnable explanatory fortress of culturally-dominant, dynamically-conventional, physicalism.)

Directists aren't solely motivated by the problem of knowledge. And even if they are, experimental support for dynamically-unconventional indirectism will not sway all minds. (For example, there's Diane's dynamically-unconventional directism, too!) Similarly, not all attacks on science are driven by post-modern intellectual critiques. Even those that are won't be ended at a stroke, by putative experimental-resolution of dynamically-conventional science's foundational issues. 
However, resolving the tangle of issues at the direct/indirect nexus - or more plausibly, establishing one comprehensive, contradiction-free, point of clarity in this locale - must have a powerful impact on the conflict between science and the postmodern school (Rosseinsky, 2021d). To the degree that it voices a single shared stance, post-modernity argues that there is no certainty, or truth. Science that hasn't resolved the direct/indirect debate, and associated problems of knowledge, can't mount a strong and effective response. (And simply ignoring or trying to dismiss the issue doesn't make to go away clearly!)

Remarkably, we are led to the view that an experimental program comparing actual-and-simulated brain-dynamics (Rosseinsky, 2021b) can resolve the dynamical character of reality, put an end to direct/indirect debates in a way that preserves both science and the capacity to know (Rosseinsky, 2021c), and thus start to bring peace to the science/postmodern conflict (Rosseinsky, 2021d).

As I suggested earlier, the ultimate 'why' for exploring the direct/indirect question is because we are interested in a peaceful, functional, society. This is the remarkable relevance of the object/representation relationship. 


\section{References}

Austin, J. L. (1962) Sense and Sensibilia, ed. G. J. Warnock. Oxford: Oxford University Press.

Ayer, A.J. (1940). The Foundations of Empirical Knowledge. New York: Macmillan.

Baldwin, J. (ed.). (2020). Navigating Post-Truth and Alternative Facts: Religion and Science as Political Theology (Religion and Science as a Critical Discourse). Lanham, MD: Lexington.

Barnes B, Bloor D, \& Henry J. (1996). Scientific Knowledge: A Sociological Analysis. Chicago, Illinois, USA: University of Chicago Press.

Blackburn, S. (1996). 'Naïve Realism', in The Oxford Dictionary of Philosophy. Oxford: Oxford University Press.

Block, N. (2005). Two neural correlates of consciousness. Trends in Cognitive Sciences, 9(2), 46-52.

Block, N. (2007). Consciousness, accessibility, and the mesh between psychology and neuroscience. Behavioral and Brain Sciences, 30(5-6), 481-499.

Bohm, D. (1951). Quantum Theory. Englewood Cliffs, NJ: Prentice-Hall.

Chalmers, D. J. (1996). The Conscious Mind: In Search of a Fundamental Theory. New York: Oxford University Press, USA.

Chalmers, D. J. (2008). 'The varieties of emergence', in P. Davies \& P. Clayton (Eds.), The ReEmergence of Emergence: The Emergentist Hypothesis from Science to Religion (pp. 244-256). Oxford: Oxford University Press.

Chalmers, D. J. (2013). How can we construct a science of consciousness? Annals of the New York Academy of Sciences, 1303(1), 25-35. https://doi.org/10.1111/nyas.12166

Cohen, M. A., \& Dennett, D. C. (2011). Consciousness cannot be separated from function. Trends in Cognitive Sciences 15, 358-364.

Crick, F., \& Koch, C. (1995). Are we aware of neural activity in primary visual cortex? Nature 375, 121-123. https://doi.org/10.1038/375121a0

Dennett, D. C. (1991). Consciousness Explained. Boston, MA: Little Brown.

Dennett D. C., \& Kinsbourne, M. (1992). Time and the Observer: the Where and When of Consciousness in the Brain. Behavioral and Brain Sciences 15:183-247. 
Doerig, A., Schurger, A., Hess, K., \& Herzog, M. H. (2019). The unfolding argument: Why IIT and other causal structure theories cannot explain consciousness. Consciousness and Cognition 72, 49-59.

Elitzur, A. C. (1989). Consciousness and the incompleteness of the physical explanation of behavior. The Journal of Mind and Behavior, 10(1), 1-20.

Fahrenfort, J. J., \& Lamme, V. A. (2012). A true science of consciousness explains phenomenology: Comment on Cohen and Dennett. Trends in Cognitive Sciences, 16(3), 138-139.

French, R. (1987). The Geometry of Visual Space, Noûs 21, 114-133.

French, R. (2018). 'A Defense of Representational Realism', in Direct versus Indirect Realism: A Neurophilosophical Debate on Consciousness, Smythies, J.R., \& French, R. (eds.). London: Elsevier.

Gamez, D. (2014). The measurement of consciousness: a framework for the scientific study of consciousness. Front. Psychol 5, 714. doi:10.3389/fpsyg.2014.00714.

Goldstein, H., Poole, C. P., \& Safko, J. L. (2001). Classical Mechanics. San Francisco: AddisonWesley.

Kastrup, B. (2018). The universe in consciousness. JCS, 25(5-6), 125-155.

Kenny, A. (1971). 'The Homunculus Fallacy', in Interpretations of Life and Mind, Grene, M.G., \& Prigogine, I. (eds.). New York: Humanities Press.

Koch, C., Massimini, M., Boly, M., \& Tononi, G. (2016). Neural correlates of consciousness: progress and problems. Nat Rev Neurosci 17, 307-321. https://doi.org/10.1038/nrn.2016.22

Latour, B. (2010). On the Modern Cult of the Factish Gods. Durham, NC: Duke University Press. Lyotard, J.-F. (1979). La Condition Postmoderne. Paris: Minuit.

McIntyre, L. (2018). Post-Truth. Cambridge, MA: MIT Press.

Melloni, L., Mudrik, L., Pitts, M., \& Koch, C. (2021). Making the hard problem of consciousness easier. Science 372 (6545), 911-912. https://doi.org/10.1126/science.abj3259.

Moore, G.E. (1953) 'Sense-Data’ in Some Main Problems of Philosophy. London: Allen Unwin.

A. Nieder, A., L.Wagener, L., \& Rinnert, P. (2020). A neural correlate of sensory consciousness in a corvid bird. Science 369 (6511) 1626-1629 (2020). https://doi.org/10.1126/science.abb1447. 
Phillips, I. (2018). The methodological puzzle of phenomenal consciousness. Philosophical Transactions of the Royal Society B: Biological Sciences, 373(1755), 20170347. https://doi.org/10.1098/rstb.2017.0347.

Putnam, H. W. (1999). The Threefold Cord: Mind, Body, and World. New York: Columbia University Press.

Rosseinsky, N. M. (2015). The measurement of consciousness: assuming completeness of first-person report significantly restricts scope and reliability of theory-discrimination. Front. Psychol. 6. https://doi.org/10.3389/fpsyg.2015.00025.

Rosseinsky, N.M. (2021a). 'The meter-Hamiltonian argument (1): conscious experience cannot be explained scientifically under current physics', Part 2 in From Godel to Trump: A Three-Step Resolution to Science's Emerging Crisis ( 'Fake News', Consciousness Science, and the Path to a postpost-Truth Society). San Diego, CA: tEoM Press.

Rosseinsky, N.M. (2021b). 'The meter-Hamiltonian argument (2): why comparing actual brain-data to computer simulations must be consciousness science's first priority', Part 3 in From Godel to Trump: A Three-Step Resolution to Science's Emerging Crisis ('Fake News', Consciousness Science, and the Path to a post-post-Truth Society). San Diego, CA: tEoM Press.

Rosseinsky, N.M. (2021c). 'The meter-Hamiltonian argument (3): reliably-scientific foundations of physics depend on existence of a specific natural dynamical-order', Part 4 in From Godel to Trump: A Three-Step Resolution to Science's Emerging Crisis ('Fake News', Consciousness Science, and the Path to a post-post-Truth Society). San Diego, CA: tEoM Press.

Rosseinsky, N.M. (2021d). From Godel to Trump: A Three-Step Resolution to Science's Emerging Crisis ('Fake News', Consciousness Science, and the Path to a post-post-Truth Society). San Diego, CA: tEoM Press.

Rosseinsky N.M. (2022a) “"Effing Consciousness” - Or, How to Use New Symbols to Get Qualia into the Hard Sciences', Chapter 2 in The Science We Need: One Experiment to Change the World. San Diego, CA: tEoM Press (2022, forthcoming). [2019 preprint: https://doi.org/10.31234/osf.io/xb3g5]

Rosseinsky N.M. (2022b) 'N-Theory: One Hypothesis to Meet It All', in The Science We Need: One Experiment to Change the World. San Diego, CA: tEoM Press (2022, forthcoming).

Rosseinsky N.M. (2022c) ‘Installing Physical Theory’s Missing Symbolism for Conscious Experience Reveals Ten Puzzles for Science as A Whole', in Towards a Real Science of Consciousness: Seven 
(Relatively!) Easy Pieces. San Diego, CA: tEoM Press (2022, forthcoming). [2018 preprint: https://doi.org/10.13140/RG.2.2.36652.31361/1.]

Rudd, A. (2000). Phenomenal judgment and mental causation. JCS, 7(6), 53-66.

Russell, B. (1912). 'Appearance and Reality', in The Problems of Philosophy. London: Williams and Norgate.

Smythies, J.R. (2003). Space, Time, and Consciousness. JCS 10(3), 47-56.

Sokal, A., \& Bricmont, J. (1999). Fashionable Nonsense: Postmodern Intellectuals'Abuse of Science. London, UK: Picador.

Stoljar, D. (2021). 'Physicalism' in The Stanford Encyclopedia of Philosophy (Summer 2021 Edition), Zalta, E.N., (ed.). https://plato.stanford.edu/archives/sum2021/entries/physicalism/.

Tsuchiya, N., Andrillon, T., \& Haun, A. (2019). A reply to 'the unfolding argument': Beyond functionalism/behaviorism and towards a truer science of causal structural theories of consciousness. https://doi.org/10.31234/osf.io/a2ms9

Vardanyan, M., Trotta, R., \& Silk, J. (2009). How flat can you get? A model comparison perspective on the curvature of the Universe. Monthly Notices of the Royal Astronomical Society. 397 (1): $431-$ 444. arXiv:0901.3354

Wagner, M. (2006). The Geometries of Visual Space. New York: Psychology Press.

Weinberg, S. (1977). The First Three Minutes: A Modern View of the Origin of the Universe. New York: Basic.

Wheeler, J.A. (1989). 'Information, physics, quantum: The search for links', in Complexity, Entropy, and the Physics of Information, Zurek, W., (ed.). Redwood City, CA: Addison-Wesley.

Wilkes, K. V. (1988). —, Yishi, , duh, um, and consciousness. In A. Marcel \& E. Bisiach (Eds.), Consciousness and Contemporary Science (pp. 16-41). Oxford: Clarendon Press.

Yaron, I., Melloni, L., \& Pitts, M. (2021). The Consciousness Theories Studies (ConTraSt) database: analyzing and comparing empirical studies of consciousness theories. https://doi.org/10.1101/2021.06.10.447863. 